\title{
Distinctive Clinical and Pathologic Features of Immature Teratomas Arising from Induced Pluripotent Stem Cell Injection in a Patient with type 2 Diabetes: A Case Report
}

\section{Lei Han}

The Third Affiliated Hospital of Kunming Medical University, Tumor Hospital of Yunnan Province $\mathrm{HaO} \mathrm{He}$

The Third Affiliated Hospital of Kunming Medical University, Tumor Hospital of Yunnan Province Yihao Yang

The Third Affiliated Hospital of Kunming Medical University, Tumor Hospital of Yunnan Province Qingyin Meng

The Third Affiliated Hospital of Kunming Medical University, Tumor Hospital of Yunnan Province Fan Ye

The Third Affiliated Hospital of Kunming Medical University, Tumor Hospital of Yunnan Province Gong Chen

The Third Affiliated Hospital of Kunming Medical University, Tumor Hospital of Yunnan Province Jing Zhang ( $\square$ nmlimit@sina.com )

The Third Affiliated Hospital of Kunming Medical University, Tumor Hospital of Yunnan Province

\section{Research Article}

Keywords: T2DM, Immature teratoma, iPSC, Clinical and pathologic features

Posted Date: July 26th, 2021

DOI: https://doi.org/10.21203/rs.3.rs-664252/v1

License: (a) (i) This work is licensed under a Creative Commons Attribution 4.0 International License. Read Full License 


\section{Abstract}

Background: As a new potential cure for diabetes, induced pluripotent stem cells (iPSCs) are characterized by self-renewal capacity and the ability to differentiate into pancreatic islet $\beta$-cells, which will hopefully secrete insulin and rebuild blood glucose balance. The safety and validity of iPSC treatment for diabetes remain controversial. One of the most serious risks is teratoma formation arising from undifferentiated stem cells, but clinical reports are rare.

Case presentation: Here, we report a distinctive case of immature teratoma after iPSC treatment for diabetes, which was accidentally treated in our soft tissue sarcoma centre. The patient received islet $\beta$ cell injection, in which the cells were differentiated from autologous iPSCs, into the deltoideus muscle. Two months later, a mass located in the injection area formed with enlarged axillary lymph nodes. Here, we present the different clinical, radiological and pathological features of this immature teratoma in detail. Distinctive from typical immature teratomas, the tumour was characterized by rapid growth and local lymph node metastasis. The tumour was not sensitive to typical chemotherapy regimens for immature teratomas. MRI scanning showed heterogeneous enhancement and a rich blood supply to the tumour. The histopathology showed immature endoderm, mesoderm and ectoderm tissues composed of osseous, cartilaginous, vascular and adenoid tissues, which have more cellular atypia than typical teratomas. Staining for both Oct-4 and Sox-2 was positive in the tumour cell nucleus by immunofluorescence assay, but insulin staining was negative. Next-generation sequencing showed many missense mutations, but abnormal gene rearrangement or copy number was not observed.

Conclusions: More attention should be given to teratoma formation after iPSC treatment for diabetes, which is more aggressive than typical teratomas. The safety and validity of iPSC treatment for diabetes should be confirmed by more standardized clinical trials.

\section{Background}

Diabetes mellitus (DM) is a type of metabolic disease characterized by hyperglycaemia, insulin resistance and defective blood glucose regulation, which can damage various organ functions. According to statistics from the World Health Organization, in 2030, the number of diabetic patients in the world will double, causing a serious socioeconomic burden [1]. Type 2 diabetes (T2DM) accounts for $85-95 \%$ of all cases and is characterized by insulin resistance in insulin-responsive tissues and impaired insulin secretion by pancreatic $\beta$-cells[2]. The majority of patients need to inject insulin for their lifetime to control blood sugar, which always results from serious complications caused by irreversible pancreatic $\beta$-cell damage [3]. In recent years, an increasing number of experts have begun to use regenerative medicine to treat irreversible pancreatic damage to $\beta$-cells in pancreatic islets using human pluripotent stem cells (iPSCs)[4]. Because autologous iPSCs are easy to obtain and expandable and can be differentiated into any desired cells, they can avoid ethical conflicts and immune rejection. iPSC technology is used in many fields and has created a new frontier, including regenerative therapy, disease modelling, drug toxicity assessment and developmental biology, and also represents a potential cure for T2DM[5, 6]. 
However, the tumourigenicity of iPSCs hinders the wide application of this technology in clinical research. It is difficult to clarify the validity and safety of iPSC treatment based on the limited number of clinical trials (i.e., NCT03403699)[7]. In in vitro and in vivo iPSC experimental models, wide genomic instability has been detected, including chromosomal aberrations, copy number variations, and single nucleotide variations $[8,9]$. A few clinical cases have reported tumour or tumour-like lesion formation after stem cell transplantation $[10,11]$. However, there are few clinical reports about tumour formation after iPSC treatment for T2DM.

Here, we report a case of immature teratoma formation after iPSC treatment for T2DM. The patient received islet $\beta$-cell injection into the deltoideus muscle, where the cells were differentiated from autologous iPSCs at another hospital. Two months later, a mass located in the injection area formed with enlarged axillary lymph nodes. The patient continued to be treated in our soft tissue sarcoma centre. Herein, we present the different clinical, radiological and pathological features of this immature teratoma in detail.

\section{Case Presentation}

\section{Initial hospital experience}

The patient was a 49-year-old male with T2DM for 17 years. The patient used $20 \mathrm{U}$ insulin degludec every morning with poor blood sugar control. He received iPSC treatment in Chennuo Medical Hospital, Beijing, China. In short, somatic cells were isolated from his own blood. The patient-derived somatic cells were reprogrammed pluripotent stem cells (PSCs). PSCs were induced into islet $\beta$-cells, which were expanded and injected into the deltoid muscle of the left upper arm. However, two months later, the blood sugar regulation and insulin levels of the patient did not improve. The patient found a mass located in the injection area with enlarged axillary lymph nodes. He felt no discomfort and visited our soft tissue sarcoma centre of Yunnan Province.

\section{Soft Tissue Sarcoma Centre of Yunnan Province}

\section{Clinical treatment}

The patient complained of a growing mass in the left upper arm without any discomfort. Physical examination showed that a solid lump in the deltoid muscle was $6 \mathrm{~cm} \times 5 \mathrm{~cm} \times 5 \mathrm{~cm}$ with hard texture, unclear boundaries, poor mobility, and no redness or inflammation. A variety of imaging examinations in the outpatient department suggested a benign or intermediate tumour. Therefore, the patient underwent total excision biopsy of the mass in the left upper arm, and the pathological diagnosis confirmed immature teratoma. Further PET-CT examinations revealed ipsilateral axillary and cervical lymph node metastasis. After routine BEP regimen (bleomycin $30 \mathrm{Ud}$, etoposide $100 \mathrm{mg} / \mathrm{m}^{2} \mathrm{~d} 1-5$ and cisplatin 20 $\left.\mathrm{mg} / \mathrm{m}^{2} \mathrm{~d} 1-5\right)$ chemotherapy for 2 cycles, the tumour progressed. Then, axillary and neck lymph node dissection was performed, which revealed 4/28 lymph node metastases with extracapsular invasion. 
Adjuvant radiotherapy (intensity modulation $50 \mathrm{~Gy}, 25$ times) was performed on the neck and axillary surgery area combined with the TIP regimen (albumin-bound paclitaxel $250 \mathrm{mg} / \mathrm{m}^{2} \mathrm{~d} 1$, ifosfamide 2 $\mathrm{g} / \mathrm{m}^{2} \mathrm{~d} 1-3$ and cisplatin $\left.30 \mathrm{mg} / \mathrm{m}^{2} \mathrm{~d} 1-5\right)$ chemotherapy for 4 cycles. To date, there has been no recurrence or distant metastasis.

\section{Imaging features}

Colour Doppler ultrasound in the outpatient department showed subcutaneous hypoechoes of 7.5 $\mathrm{cm} \times 6.7 \mathrm{~cm} \times 5.1 \mathrm{~cm}$ with an intermediate blood supply. An X-ray scan showed a soft tissue shadow with no clear bone destruction of the humerus (Fig. 1a). A plain CT scan showed a round, mixed-density mass in the deltoid muscle with an unclear boundary (Fig. 1b). An enhanced CT scan showed uneven and moderate enhancement (Fig. 1C), which indicated benign or intermediate tumours in the diagnosis. Plain MRI scan showed iso-signal on T1W imaging and high-signal on T2W imaging of the soft tissue mass in the deltoid muscle and enlarged axillary lymph nodes, both of which were uneven and obviously enhanced in enhanced MRI scan (Fig. 1d, e). After excision biopsy of the mass, a PET/CT scan showed ipsilaterally axillary, supraclavicular and neck lymph node metastasis with a maximum diameter of 2.1 $\mathrm{cm}\left(\mathrm{SUV}_{\max }\right.$ 5.4). There were no abnormalities in the whole body, including the testis.

\section{Pathological features}

The histopathology of the soft tissue tumour of the left upper arm showed immature endoderm, mesoderm and ectoderm tissues composed of osseous, cartilaginous, vascular and adenoid tissues, which had more cellular atypia than typical teratomas (Fig. 2a). Lymph node dissection specimens showed tumour metastasis (left axillary $2 / 3$, left apical lymph node $0 / 4$, left thoracic intermuscular lymph node $1 / 1$, left supraclavicular lymph node $1 / 11$ and left cervical root lymph node $0 / 9$ positive with extracapsular invasion) (Fig. 2b). Postoperative immunohistochemistry of the left axillary mass (X202016410) showed CK (-), Vim (+), Ki-67 (approximately 15\%) Actin (part +) SMA (part +) Des (part +) S-100 (-) CD10 (part +) ER (-) CD34 (blood vessel +) Syn (small cell +) CgA (-). To confirm iPSC differentiation, the key markers of Oct4 and Sox 2 gene amplification were detected by immunofluorescence assay, both of which were positive in the nucleus (Fig. 2c, d). However, immunohistochemical staining of insulin was negative in tumour cells.

Next-generation sequencing (NGS) of tumour tissues showed 16 gene missense or frameshift mutations, including ABCB4, CDC73, CROT, DNMT3A, HCK, KMT2D, MSH2, MSH6, NCOR2, PRDM1, PREX2, RPS6KB2, TRAF7, ZFHX3, ZNF703 and ZRSR2. However, there was no gene arrangement or copy number variation. The genetic test results did not match the potentially beneficial targeted drugs. The percentage of PD-L1-positive cells was 1-2\% in tumour tissue. The mismatch repair (MMR) assay by immunohistochemistry showed proficiency (pMMR).

\section{Discussion And Conclusions}


IPSC treatment has obtained amazing effects in preliminary clinical trials of immune or neurodegenerative disease [12, 13]. It also provides a new treatment model for T2DM [14]. Compared with other stem cell treatments, iPSCs have the advantages of easy acquisition, rapid expansion, low immunogenicity, and no ethical issues [15]. However, tumourigenicity is the greatest risk of iPSC treatment, which blocks its further application $[16,17]$.

Increasing evidence shows that reprogramming of iPSCs carries the risk of tumourigenesis, such as genome insertion of genetic recombination vectors, overexpression of oncogenic transcription factors, and overall hypomethylation similar to cancer[18]. In addition to the inherent tumourigenic risk induced by pluripotency, a long-term culture environment produces genomic abnormalities, and the final result is that the propagation of cultures has stable and heterogeneously acquired tumourigenic potential[19]. In addition, it is necessary to strike a balance between tumourigenicity and immunogenicity to obtain complete and mature pancreatic $\beta$-cells to prevent the occurrence of teratomas.

Although there are few clinical trials on tumour formation after stem cell transplantation, it is still uncertain whether a real tumour is formed. We firstly reported a clinical case of immature teratoma formed after iPSC treatment in T2DM. Unlike mature teratomas, immature teratomas have the potential for recurrence and metastasis. Therefore, they can be localized anywhere except in the limbs and lymph nodes[20]. Pashankar $\mathrm{F}$ et al. have shown that postoperative chemotherapy is standard in immature teratoma disease[21]. The tumour in the left upper arm developed rapid growth and local lymph node metastasis but was insensitive to chemotherapy. Okur FV et al. found that all iPSC lines expressed pluripotency-related genes, and disease-related mutations were retained in patient-specific iPSCs[22]. However, there was more cell atypia than in typical teratomas, which meant an increase in the components. The positive Oct4 and Sox 2 indicators proved that the injected cells were not fully differentiated, and the negative insulin showed no insulin secretion function, which indicated that the differentiation state induced by iPSCs in vitro does not represent the state after implantation in vivo. A standardized good manufacturing practices (GMP) protocol strengthens the effective supervision and standardization of cell production processes to produce pancreatic $\beta$-cells and improves the effectiveness and safety of iPSC treatment[23].

Although iPSCs initiated the dawn of diabetes treatment, their tumourigenicity seriously hindered their clinical application and expansion. To the best of our knowledge, there is no clinical practice guidance for the appropriate treatment of teratomas caused by iPSC injection. We chose chemotherapy regimens in accordance with highly aggressive sarcoma[24]. Although radiation therapy is sometimes used in addition to surgery and chemotherapy, its benefits are unclear[25]. In this case, combined with the relevant genetic test results, we consider that the tumour is not sensitive to immunotherapy. At present, a large number of researchers are working on developing new reprogramming methods or establishing new mutation screening procedures (such as introducing non-tumourigenic genes and viruses) and adopting more standardized GMP production processes (such as 3D culture) to avoid tumour formation and immune response after transplantation[26]. In addition, vaccines based on iPSCs may provide a new 
cancer immunotherapy option, but their efficacy and related immune mechanisms still need to be further studied and confirmed[27].

Based on the few clinical trials for T2DM currently available, the safety and validity of iPSC treatment need to be confirmed by more standardized clinical trials. More attention should be given to teratoma formation after iPSC treatment for T2DM, which is more aggressive than typical teratomas.

\section{Abbreviations}

iPSCs: induced pluripotent stem cells; MRI: Magnetic Resonance Imaging; OCT4: Recombinant Octamer Binding Transcription Factor 4; Sox-2: Octamer binding factor 4; GMP: Good Manufacturing Practice; DM: Diabetes mellitus; T2DM: Type 2 Diabetes Mellitus; BEP: bleomycin,etoposide and cisplatin; TIP: albuminbound paclitaxel ifosfamide and cisplatin; PET/CT囚 Positron Emission Tomography/Computed Tomography; SUV囚 standard uptake value; PD-1囚 programmed death 1.

\section{Declarations}

\section{Acknowledgements}

We wish to thank Jie He (920th Hospital of Joint Logistics Support Force of the Chinese People's Liberation Army, Kunming, China) for her advice and assistance in immunofluorescent staining of Oct-4 and Sox-2.

\section{Authors' contributions}

Lei Han and Hao He were major contributors to data collection and the writing of the manuscript. Yihao Yang completed immunohistochemistry assay. Qingyin Meng completed immunofluorescence assay. Fan Ye and Gong Chen contributed to Figures collection. Jing Zhang designed the main treatment plan and revised the manscript. All authors have read and approved the final manuscript.

\section{Funding}

This work was supported by the Top Young Talents, Ten Thousand People Plan of Yunnan province (no.YNWR-QNBJ-2019-163) and Applied Basic Research Foundation of Yunnan Province (no.2019FE001074) .

\section{Availability of data and materials}

All data generated or analyzed during this study are included in this published article. 


\section{Competing interests}

All authors declare that they have no competing interests.

\section{Consent for publication}

Consent was obtained from the patient to allow their information and images to be published.

\section{Ethics approval and consent to participate}

Treatment in tumor hospital of Yunnan province: Consent was obtained. A case report does not require approval from our institutional Research Board.

\section{Author details}

${ }^{1}$ Department of Orthopaedics, Bone and Soft Tissue Tumors Research Center of Yunnan Province, The Third Affiliated Hospital of Kunming Medical University, Tumor Hospital of Yunnan Province, Kunming, Yunnan 650118, P.R. China. ${ }^{2}$ Department of Pathology, The Third Affiliated Hospital of Kunming Medical University, Tumor Hospital of Yunnan Province, Kunming, Yunnan 650118, P.R. China.

\section{References}

1. Saeedi P, Petersohn I, Salpea P, Malanda B, Karuranga S, Unwin N, et al. Global and regional diabetes prevalence estimates for 2019 and projections for 2030 and 2045: Results from the International Diabetes Federation Diabetes Atlas, 9(th) edition. Diabetes Res Clin Pract. 2019;157:107843.

2. Keane KN, Calton EK, Carlessi R, Hart PH, Newsholme P. The bioenergetics of inflammation: insights into obesity and type 2 diabetes. Eur J Clin Nutr. 2017;71(7):904-12.

3. Gurzov EN, Ke PC, Ahlgren U, Garcia Ribeiro RS, Gotthardt M. Novel Strategies to Protect and Visualize Pancreatic beta Cells in Diabetes. Trends Endocrinol Metab. 2020;31(12):905-17.

4. Hogrebe NJ, Augsornworawat P, Maxwell KG, Velazco-Cruz L, Millman JR. Targeting the cytoskeleton to direct pancreatic differentiation of human pluripotent stem cells. Nat Biotechnol. 2020;38(4):46070 .

5. Chang PH, Chao HM, Chern E, Hsu SH. Chitosan 3D cell culture system promotes naive-like features of human induced pluripotent stem cells: A novel tool to sustain pluripotency and facilitate differentiation. Biomaterials. 2021;268:120575.

6. Shi Y, Inoue H, Wu JC, Yamanaka S. Induced pluripotent stem cell technology: a decade of progress. Nat Rev Drug Discov. 2017;16(2):115-30. 
7. Wu Q, Zheng S, Qin Y, Zheng X, Chen H, Yang T, et al. Efficacy and safety of stem cells transplantation in patients with type 1 diabetes mellitus-a systematic review and meta-analysis. Endocr J. 2020;67(8):827-40.

8. Rohani L, Johnson AA, Naghsh P, Rancourt DE, Ulrich H, Holland H. Concise Review: Molecular Cytogenetics and Quality Control: Clinical Guardians for Pluripotent Stem Cells. Stem Cells Transl Med. 2018;7(12):867-75.

9. Pessoa LVF, Bressan FF, Freude KK. Induced pluripotent stem cells throughout the animal kingdom: Availability and applications. World J Stem Cells. 2019;11(8):491-505.

10. Amariglio N, Hirshberg A, Scheithauer BW, Cohen Y, Loewenthal R, Trakhtenbrot L, et al. Donor-derived brain tumor following neural stem cell transplantation in an ataxia telangiectasia patient. PLoS Med. 2009;6(2):e1000029.

11. Thirabanjasak D, Tantiwongse K, Thorner PS. Angiomyeloproliferative lesions following autologous stem cell therapy. J Am Soc Nephrol. 2010;21(7):1218-22.

12. Doi D, Magotani H, Kikuchi T, Ikeda M, Hiramatsu S, Yoshida K, et al. Pre-clinical study of induced pluripotent stem cell-derived dopaminergic progenitor cells for Parkinson's disease. Nat Commun. 2020;11(1):3369.

13. Bloor AJC, Patel A, Griffin JE, Gilleece MH, Radia R, Yeung DT, et al. Production, safety and efficacy of iPSC-derived mesenchymal stromal cells in acute steroid-resistant graft versus host disease: a phase I, multicenter, open-label, dose-escalation study. Nat Med. 2020;26(11):1720-5.

14. Meng QS, Liu J, Wei L, Fan HM, Zhou XH, Liang XT. Senescent mesenchymal stem/stromal cells and restoring their cellular functions. World J Stem Cells. 2020;12(9):966-85.

15. Felipe A, Ferrer-Montiel A. The Spanish Ion Channel Initiative (SICI) Consortium: Ten Years (2008(-)2018) of a Network of Excellence on Ion Channel Research. Int J Mol Sci. 2018;19(11).

16. Ortmann D, Vallier L. Variability of human pluripotent stem cell lines. Curr Opin Genet Dev. 2017;46:179-85.

17. Liu QW, Huang QM, Wu HY, Zuo GS, Gu HC, Deng KY, et al. Characteristics and Therapeutic Potential of Human Amnion-Derived Stem Cells. Int J Mol Sci. 2021;22(2).

18. Lee AS, Tang C, Rao MS, Weissman IL, Wu JC. Tumorigenicity as a clinical hurdle for pluripotent stem cell therapies. Nat Med. 2013;19(8):998-1004.

19. Mayshar Y, Ben-David U, Lavon N, Biancotti JC, Yakir B, Clark AT, et al. Identification and classification of chromosomal aberrations in human induced pluripotent stem cells. Cell Stem Cell. 2010;7(4):521-31.

20. Hassan HM, Omar AS, Mohammed AA. Mature cystic teratoma of the neck misdiagnosed at cystic hygroma; Case report. Int J Surg Case Rep. 2021;78:321-5.

21. Pashankar F, Hale JP, Dang H, Krailo M, Brady WE, Rodriguez-Galindo C, et al. Is adjuvant chemotherapy indicated in ovarian immature teratomas? A combined data analysis from the Malignant Germ Cell Tumor International Collaborative. Cancer. 2016;122(2):230-7. 
22. Okur FV, Cevher I, Ozdemir C, Kocaefe C, Cetinkaya DU. Osteopetrotic induced pluripotent stem cells derived from patients with different disease-associated mutations by non-integrating reprogramming methods. Stem Cell Res Ther. 2019;10(1):211.

23. Katayama M, Hirayama T, Horie K, Kiyono T, Donai K, Takeda S, et al. Induced Pluripotent Stem Cells With Six Reprogramming Factors From Prairie Vole, Which Is an Animal Model for Social Behaviors. Cell Transplant. 2016;25(5):783-96.

24. Donadio AC, Motzer RJ, Bajorin DF, Kantoff PW, Sheinfeld J, Houldsworth J, et al. Chemotherapy for teratoma with malignant transformation. J Clin Oncol. 2003;21(23):4285-91.

25. Hackethal A, Brueggmann D, BohImann MK, Franke FE, Tinneberg HR, Munstedt K. Squamous-cell carcinoma in mature cystic teratoma of the ovary: systematic review and analysis of published data. Lancet Oncol. 2008;9(12):1173-80.

26. Wang Y, Yuan F, Wang A, Wang C, Lin L, Wang S, et al. Induced pluripotent stem cells (SHCDNi002-A cells) isolated from the peripheral blood mononuclear cells of a 1-year-old Chinese girl with mediator complex subunit 12-related syndrome. Stem Cell Res. 2020;46:101838.

27. Goyvaerts C, Breckpot K. Towards a personalized iPSC-based vaccine. Nat Biomed Eng. 2018;2(5):277-8.

\section{Figures}

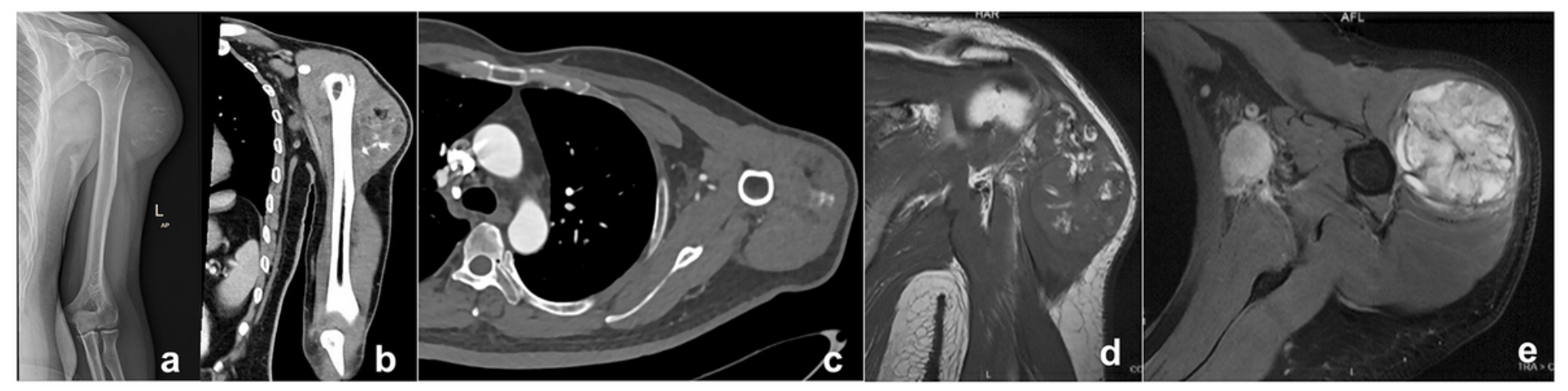

\section{Figure 1}

Imaging features before treatment. a X-ray image. In the deltoid muscle of the left upper arm, a round, mixed-density mass with an unclear boundary of $9.6 \mathrm{~cm} \times 5.2 \mathrm{~cm}$ was observed. The humeral cortex is intact. b, c Plain CT scan showed a round, mixed-density mass in the deltoid muscle with an unclear boundary. An enhanced CT scan showed uneven and moderate enhancement. d-e MRI T1W and T2W images. T1W was dominated by iso-signals, with patchy high- and low-signal areas. In addition, T2W1 also showed high-signal and patchy low-signal areas. The left axilla had multiple lymph node swelling, the signal was fair on plain scan, and the enhancement was roughly uniform. 

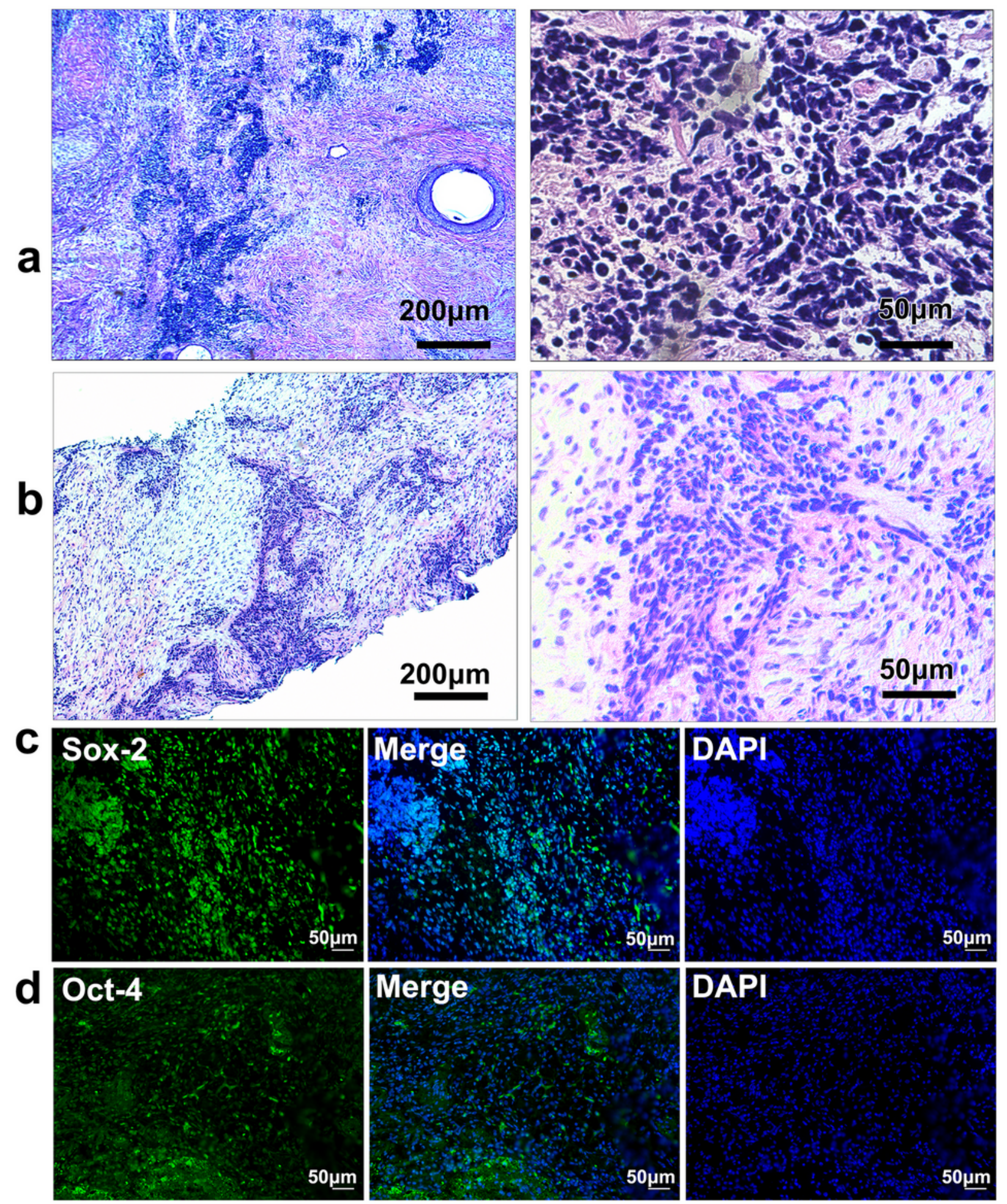

Figure 2

Pathology before and after treatment. a HE staining of the left upper arm tumour. Bone, cartilage, vasculature and adenoid tissue and other components, as well as the neuropillar structure and small round cells distributed in small pieces. The various components are arranged in a disorderly manner, and the shape of the lesion conforms to an immature teratoma, grade II. b HE staining of metastatic axillary lymph node. Different immature teratoma cells mixed in among small round lymphocytes. c-d 
Immunofluorescence staining of iPSC differentiation markers. Both Oct-4 and Sox-2 staining showed nuclear positivity in paraffin sections of the left upper arm tumour. 